\title{
STABILIZATION IN A GRADIENT SYSTEM WITH A CONSERVATION LAW
}

\author{
ROBERT L. PEGO \\ (Communicated by Kenneth R. Meyer)
}

\begin{abstract}
Suppose $\sum \mu_{j}=1$ and $F: \mathbf{R} \mapsto \mathbf{R}$ is $C^{1}$ with $F^{\prime}$ piecewise $C^{1}$. For the finite system of ordinary differential equations

$$
\dot{u}_{i}=F^{\prime}\left(u_{i}\right)-\sum_{j} \mu_{j} F^{\prime}\left(u_{j}\right)=0,
$$

I prove that every bounded solution stabilizes to some equilibrium as $t \rightarrow$ $\infty$. For this system, $\sum \mu_{j} u_{j}$ is conserved and the quantity $\sum \mu_{j} F\left(u_{j}\right)$ is nonincreasing and serves as a Lyapunov function, but the set of equilibria can be connected and degenerate. Essential use is made of a result related to one of Hale and Massat that an $\omega$-limit set that lies in a $C^{1}$ hyperbolic manifold of equilibria must be a singleton.
\end{abstract}

\section{INTRODUCTION}

Given a function $F: \mathbf{R} \mapsto \mathbf{R}$ with locally Lipschitz derivative $f=F^{\prime}$, the equation of gradient flow for the potential $F$ is $\dot{u}+f(u)=0$. Trivially, any bounded solution $u: \mathbf{R}^{+} \mapsto \mathbf{R}$ of this equation must stabilize (converge as $t \rightarrow \infty)$ to some critical point, a point where $f=0$. Suppose we allow $u$ to depend on a parameter $x$ that lies in a probability space $\Omega$. Denote the expected value of a measurable function $g: \Omega \mapsto \mathbf{R}$ by $\int g$ or $\int g(x) d x$. Now consider the equation of gradient flow for the functional $I(u)=\int F(u(x, t)) d x$ subject to the constraint that $\int u(x, t) d x$ be preserved. This equation takes the form

$$
\dot{u}(x, t)+f(u(x, t))-\int f(u(y, t)) d y=0 .
$$

The initial value problem for (1), given bounded measurable initial conditions

$$
u(x, 0)=u_{0}(x), \quad x \in \Omega,
$$

is always well posed locally in time on the Banach space $X=L^{\infty}(\Omega)$ (or better, $X=B(\Omega)$, the space of bounded measurable functions on $\Omega$, not equivalence classes), yielding a solution $u$ in $C^{1}([0, T], X)$ for some $T>0$. A simple

Received by the editors October 7, 1990.

1980 Mathematics Subject Classification (1985 Revision). Primary 34D05, 35B40.

This work was partially supported by the NSF. 
sufficient condition for the global existence of a bounded solution for $t>0$ is that for all $u \in \mathbf{R}$,

$$
\liminf _{z \rightarrow-\infty} f(z)<f(u)<\limsup _{z \rightarrow \infty} f(z),
$$

which is easily shown as in [NP]. The question I would like to answer is: Must every bounded solution of (1) stabilize? In this paper I will demonstrate the affirmative in the following case, which corresponds to the case when the initial data $u(x, 0)$ take only a finite nuimber of values.

Theorem 1. Suppose $\Omega$ is a finite set and $f$ is $C^{1}$ or is locally Lipschitz and piecewise $C^{1}$. If $u: \Omega \times \mathbf{R}^{+} \mapsto \mathbf{R}$ is a bounded solution of $(1)$, then $\lim _{t \rightarrow \infty} u(\cdot, t)$ exists.

At first, this result seems only too obvious: Multiply (1) by $\dot{u}$ and integrate over $\Omega \times[0, t]$. One obtains the dissipation identity

$$
\int F(u(x, t)) d x+\int_{0}^{t} \int \dot{u}(x, s)^{2} d x d s=\int F(u(x, 0)) d x .
$$

Since $\int F(u(x, t)) d x$ is nonincreasing and bounded, it converges as $t \rightarrow \infty$. One can check that $\alpha(t)=\int \dot{u}(x, t)^{2} d x=\int\left(f(u)-\int f(u)\right)^{2}$ is Lipschitz. Hence since $\int_{0}^{\infty} \alpha(t) d t<\infty$, it follows $\alpha(t) \rightarrow 0$ as $t \rightarrow \infty$. This certainly implies that (1) can have no time-periodic solutions and rules out many other types of recurrent nonequilibrium behavior. If some solution of (1) does fail to stabilize, it will have to wander ever more slowly as $t \rightarrow \infty$. One suspects that, in fact, a definite limit must always be attained.

Here the going becomes difficult when $\Omega$ is infinite, so assume $\Omega$ is finite, $\Omega=\{1, \ldots, N\}$. A point $u \in \mathrm{L}^{\infty}(\Omega)$ may be identified with the point, $(u(1), \ldots, u(N))^{T} \in \mathbf{R}^{N}$, and $\int g=\sum \mu_{i} g(i)$ for some positive constants $\mu_{i}$ with $\sum_{i=1}^{N} \mu_{i}=1$. The $\omega$-limit set of the solution in $\mathbf{R}^{N}$ is nonempty, compact, and connected; denote it by $\omega_{0}$. As a consequence of the above, the $\omega$-limit set is contained in the set of equilibria $E$, and we have, for $u$ in $\omega_{0}$,

$$
f(u(x)) \equiv \int f(u), \quad \int F(u)=c_{1}, \quad \int u=c_{0}
$$

for constants $c_{0}, c_{1}$ independent of $u$.

Denote the set of $u$ in $\mathbf{R}^{N}$ satisfying (4) by $E_{c}$. At this point, if one imposes certain additional restrictions on $f$, it follows that the connected components of $E_{c}$ are singletons, so stabilization follows. For example, if $f$ is monotone, this is trivial. Andrews and Ball [AB], in a related stabilization problem for a nonlinear partial differential equation, introduced an assumption they called a "nondegeneracy condition." Assume that $f$ is a piecewise monotone, and in particular, that

for $z$ in any bounded set of $\mathbf{R}$, the equation $f(z)=s$ has a finite number $M=M(s)$ of roots $z_{1}(s)<z_{2}(s)<\cdots<z_{M}(s)$, where $M(s)$ is piecewise continuous, jumping a finite number of times.

The nondegeneracy condition of Andrews and Ball asserts that the derivatives $z_{j}^{\prime}(s), j=1, \ldots, M$ are linearly independent on any common interval of definition. This is equivalent to the following condition, which we call condition (AB): 
The functions $1, z_{1}(s), \ldots, z_{M}(s)$ are linearly independent functions of $s$ on any interval where $M(s)$ is constant.

If condition (AB) holds, stabilization follows. Any nontrivial connected component of $E_{c}$ must also lie in $E$ and, if not a singleton, must contain a curve $u(s)$ parametrized by $s=\int f(u)$, so that

$$
u_{i}(s)=z_{j(i)}(s), \quad i=1, \ldots, N, s \text { in an open interval } .
$$

Then the requirement of conservation, that $\sum \mu_{i} z_{j(i)}(s)=c_{0}$, is incompatible with the condition $(\mathrm{AB})$. Hence $E_{c}$ is totally disconnected and stabilization follows.

Condition $(\mathrm{AB})$ is rather unsatisfactory, however, since it fails to hold in many basic cases. For example, condition (AB) is always violated when $f$ is nonmonotone and piecewise linear. As another example, take $f$ to be a cubic polynomial, $f(z)=z^{3}-z$. Then $z_{1}(s)+z_{2}(s)+z_{3}(s)=0$ for $|s|<2 / 3 \sqrt{3}$. An arbitrary function $f$ can always be perturbed locally so that on some interval in $s$, condition (AB) fails.; so it seems desirable to develop arguments that can establish stabilization without the hypothesis (AB). Such arguments could be relevant in problems related to that considered by Andrews and Ball, such as have been considered in [P, NP, NPT].

If condition (AB) fails to hold, the set $E_{c}$ of equilibria that satisfy the constraint in (4) may contain nontrivial connected components; so one cannot a priori rule out the possibility that the $\omega$-limit set fails to be a singleton. The main tool used in this paper to rule out this possibility, under the hypotheses of Theorem 1, is basically due to Hale and Massat [HM]. A slight modification suitable for use in the present context is

Theorem 2. Consider the system of ordinary differential equations in $\mathbf{R}^{N}$ given by

$$
\dot{U}=G(U) \text {. }
$$

Suppose this system admits a manifold $\mathscr{M}$ of equilibria such that $G$ is $C^{1}$ in a neighborhood of $\mathscr{M}$ and $\mathscr{M}$ is hyperbolic. If the $\omega$-limit set of some trajectory contains a point of $\mathscr{M}$, but the trajectory does not stabilize, then the $\omega$-limit set contains points that are not equilibria.

The proof will be given in the appendix. Recall that a manifold of equilibria $\mathscr{M}$ is hyperbolic if for each $U$ in $\mathscr{M}$ the linearization $G^{\prime}(U)$ has no eigenvalues with zero real part other than $\lambda=0$, and $\operatorname{ker} G^{\prime}(U)^{2}=\operatorname{ker} G^{\prime}(U)=$ the tangent space to $\mathscr{M}$ at $U$.

\section{Stabilization of MEAN force}

The proof of Theorem 1 will involve two main steps. In this section I will show that

$$
\lim _{t \rightarrow \infty} \int f(u(x, t)) d x \text { exists. }
$$

It follows that for some constant $c_{3}$, each point $u$ in the $\omega$-limit set $\omega_{0}$ satisfies

$$
\int f(u)=c_{3} \text {. }
$$


If $f$ has the additional property that for all $s \in \mathbf{R}$, the solution set of $f(z)=s$ is totally disconnected, it follows immediately that the set of $u$ in $\mathbf{R}^{N}$ satisfying (4) and (6) is totally disconnected, so $\omega_{0}$ must be a singleton. The degenerate case when the graph of $f$ has flat portions will be considered in $\S 3$ and constitutes the second main step in the proof of Theorem 1.

To prove (5), argue as follows. Suppose $|u(x, t)| \leq R$ for all $x \in \Omega, t \geq 0$. As a consequence of Sard's theorem, the set of regular values of $f$ on $[-R, R]$ is open and dense. Let

$$
f_{-}=\liminf _{t \rightarrow \infty} \int f(u(\cdot, t)), \quad f_{+}=\limsup _{t \rightarrow \infty} \int f(u(\cdot, t)) .
$$

Suppose (5) fails to hold, so that $f_{-}<f_{+}$. Then there is an open interval $J \subset\left(f_{-}, f_{+}\right)$containing no critical values of $f$ so that for $s \in J$, there are a fixed finite number of solutions in $[-R, R]$ of $f(z)=s$, which we denote by $z_{1}(s), \ldots, z_{M}(s)$. These solutions are $C^{1}$ functions of $s$ for $s \in J$.

Fixing some $s_{0} \in J$, we may extract a converging subsequence $u\left(\cdot, t_{n}\right)$ such that for each $i$, and some $j(i), u\left(i, t_{n}\right) \rightarrow z_{j(i)}\left(s_{0}\right)$ as $n \rightarrow \infty$. Define the $C^{1}$ curve $u^{*}: J \mapsto \mathbf{R}^{N}$ by $u_{i}^{*}(s)=z_{j(i)}(s)$. We have $u^{*}\left(s_{0}\right) \in \omega_{0}$, and all equilibria in a neighborhood of $u^{*}\left(s_{0}\right)$ lie on the curve $u^{*}$. Because $\omega$ is connected, it follows that, replacing $J$ by a subinterval if necessary, the curve $u^{*}$ lies in $\omega_{0}$. By (4), then, $u^{*}(s)$ is contained in the hyperplane $X=\left\{u \in \mathbf{R}^{N} \mid \sum \mu_{j} u_{j}=c_{0}\right\}$, which is an invariant hyperplane under the evolution equation (1).

Lemma 1. The curve $u^{*}$ is a hyperbolic curve of equilibria in the invariant hyperplane $X$.

Proof. I need to show that for the linearization of (1) about any point on $u^{*}$, restricted to the tangent space of $X$, the only eigenvalue with zero real part is $\lambda=0$, which is simple, with eigenvector $d u^{*} / d s$. Setting $\mu=\left(\mu_{1}, \ldots, \mu_{N}\right)^{T}$, the tangent space to $X$ is the subspace $\mu^{\perp}=\left\{u \in \mathbf{R}^{N} \mid \mu^{T} u=0\right\}$. Define $f_{i}=f^{\prime}\left(u_{i}^{*}(s)\right)$. The eigenvalue problem is to find $\lambda$ and a vector $v$ so that

$$
\left(f_{i}-\lambda\right) v-\sum_{j} \mu_{j} f_{j} v_{j}=0 \quad \text { for } i=1, \ldots, N .
$$

Reordering indices if necessary, we may assume that $f_{1} \leq f_{2} \leq \cdots \leq f_{N}$. The eigenvalue problem may be analyzed completely: Define the diagonal matrix $D=\operatorname{diag}\left\{f_{1}, \ldots, f_{N}\right\}$. For $v_{j}=1 /\left(f_{j}-\lambda\right),(7)$ reads

$$
1-\sum \mu_{j} \frac{f_{j}}{f_{j}-\lambda}=\sum \mu_{j}\left(1+\frac{f_{j}}{\lambda-f_{j}}\right)=-\lambda g(\lambda) .
$$

$g(\lambda)$ is increasing for $\lambda \in \mathbf{R} \backslash\left\{f_{1}, \ldots, f_{N}\right\}$ with

$$
g(\lambda) \rightarrow \begin{cases}\infty & \text { for } \lambda \rightarrow f_{i}-0 \\ -\infty & \text { for } \lambda \rightarrow f_{i}+0\end{cases}
$$

Lemma 2. The $N-1$ eigenvalues of (7) restricted to $\mu^{\perp}$ lie in two classes:

1. For each value $f_{i}$ that appears in the list $\left(f_{1}, \ldots, f_{N}\right)$ with multiplicity $k \geq 2, \lambda=f_{i}$ is an eigenvalue of (7) with multiplicity $k-1$ and eigenspace equal to $\operatorname{ker}(D-\lambda) \cap \mu^{\perp}$, which has dimension $k-1$.

2. For each $i$ with $f_{i}<f_{i+1}$, the unique root of $g(\lambda)=\sum \mu_{j} /\left(f_{j}-\lambda\right)$ in $\left(f_{i}, f_{i+1}\right)$ is an eigenvalue with eigenvector $v$ in $\mu^{\perp}$ given by $v_{j}=1 /\left(f_{j}-\lambda\right)$. 
Moreover, the Nth eigenvalue of (7) in $\mathbf{R}^{N}$ is $\lambda=0$, with eigenvector $v_{j}=$ $1 / f_{j}$ if $g(0) \neq 0$; when $g(0)=0, \lambda=0$ is a double eigenvalue with generalized eigenvector $v_{j}=1 / f_{j}^{2}$ satisfying

$$
f_{i} v_{i}=\sum \mu_{j} f_{j} v_{j}=1 / f_{i}
$$

and $\mu^{T} v>0$.

The proof is omitted. Now, as a consequence of $f\left(z_{j}(s)\right)=s$, we have $d u_{j}^{*} / d s=1 / f_{j}$. Hence since $u^{*}$ lies in $X, \sum \mu_{j} / f_{j}=0$, and we are in the case $g(0)=0$. In this case, the root given in case 2 above for that $i$ with $f_{i}<0<f_{i+1}$ is $\lambda=0$, and Lemma 2 implies exactly that this is a simple eigenvalue for (7) restricted to $\mu^{\perp}$. This proves Lemma 1 .

To finish the proof of (5), suppose it fails. Then one may apply Theorem 2 at any point on the curve $u^{*}$, to conclude that $u(\cdot, t)$ converges at $t \rightarrow \infty$. This contradicts the assumption that (5) fails. Hence (5) holds.

\section{The DEgenerate CASE}

Since (5) holds, let $S=\lim _{t \rightarrow \infty} \int f(u(x, t)) d x$ be fixed. Suppose for contradiction that $u(\cdot, t)$ fails to converge. Define

$$
a_{i}=\liminf _{t \rightarrow \infty} u(i, t), \quad b_{i}=\limsup _{t \rightarrow \infty} u(i, t) .
$$

It follows from (1) and $\dot{u} \rightarrow 0$ that $f(z)=S$ for $z \in\left[a_{i}, b_{i}\right]$ for each $i$. The strategy of the proof is to use the synchrony implied by (1) when $f\left(u_{i}\right)=S$ for several $i$ to produce points in the $\omega$-limit set that violate the constraint $\int u=c_{0}$ implied by the conservation law.

For $u$ in $\mathbf{R}^{N}$, let \# $u$ be the number of indices $i$ such that $u_{i}$ lies in the interior of $\left(a_{i}, b_{i}\right)$. Because the points in $\left[a_{1}, b_{1}\right] \times \cdots \times\left[a_{N}, b_{N}\right]$ with $\# u=0$ are isolated, we may suppose there is some $w$ in $\omega_{0}$ with $\# w>0$. Choose $w \in \omega_{0}$ so that \# $w$ is maximal. Rearranging indices, we may assume $a_{i}<w<b_{i}$ for $i=1, \ldots, K$ and $w_{i} \notin\left(a_{i}, b_{i}\right)$ for $i>K$, where $K=\# w$. Choose $t_{n} \rightarrow \infty$ so that $u\left(\cdot, t_{n}\right) \rightarrow w$ as $n \rightarrow \infty$. From (1) it follows that whenever $u(\cdot, t)$ satisfies $a_{i}<u_{i}<b_{i}$ for $i=1, \ldots, K$, then

$$
\dot{u}_{i}=\int f(u)-S=\dot{u}_{j} \quad \text { for } i, j=1, \ldots, K .
$$

Fix $\varepsilon>0$ so small that

$$
\left|u_{i}-w_{i}\right| \leq 2 \varepsilon \text { implies } a_{i}<u_{i}<b_{i} \quad \text { for } i=1, \ldots, K .
$$

For each $n$, choose $T_{n}$ maximal so that

$$
\left|u_{i}(t)-u_{i}\left(t_{n}\right)\right| \leq \varepsilon \quad \text { for } i=1, \ldots, K, t_{n} \leq t<T_{n} .
$$

Together with (9), (11) implies that $T_{n}$ is finite for each $n$, and (10) implies that for each $n$ there is a fixed choice of sign so that

$$
u_{i}\left(T_{n}\right)-u_{i}\left(t_{n}\right)= \pm \varepsilon \quad \text { for each } i=1, \ldots, K \text {. }
$$

Define $Q=\bigcup_{n=1}^{\infty}\left[t_{n}, T_{n}\right]$ 
Lemma 3.

$$
\lim _{\substack{t \rightarrow \infty \\ t \in Q}} u_{i}(t)=w_{i} \quad \text { exists for each } i>K .
$$

Proof. Since $\lim u_{i}\left(t_{n}\right)=w_{i}$, this is the only possible limit. Suppose a limit does not exist, so that for some $j$ with $K<j$,

$$
a_{j} \leq \liminf _{\substack{t \rightarrow \infty \\ t \in Q}} u_{j}(t)<\limsup _{\substack{t \rightarrow \infty \\ t \in Q}} u_{j}(t) \leq b_{j}
$$

Again, since $\lim u_{j}\left(t_{n}\right)=w_{j}$ and $u_{j}$ is continuous on $\left[t_{n}, T_{n}\right]$, we may find a subsequence $\tau_{n} \rightarrow \infty$ so that $\lim u\left(\cdot, \tau_{n}\right)$ exists with $a_{j}<\lim u_{j}\left(\tau_{n}\right)<b_{j}$. Then (11), (12) imply $a_{i}<\lim u_{i}\left(\tau_{n}\right)<b_{i}$ for $i=1, \ldots, K$, contradicting the fact that $K=\# w$ was chosen to be maximal. This proves the lemma.

To finish the proof, we choose a subsequence of $T_{n}$ so that $u\left(\cdot, T_{n}\right)$ converges to some $w^{*}$ as $n \rightarrow \infty$. By (13) and Lemma 3, for a fixed choice of sign we have

$$
w_{i}^{*}= \begin{cases}\omega_{i} \pm \varepsilon & \text { for each } i=1, \ldots, K, \\ w_{i} & \text { for } i>K .\end{cases}
$$

Then $w$ and $w^{*}$ both lie in $w_{0}$, while $\int w \neq \int w^{*}$. This contradicts (4) and finishes the proof of Theorem 1.

\section{APPENDIX}

Proof of Theorem 2. By translation we may assume $0 \in \mathscr{M}$ lies in the $\omega$-limit set of some trajectory. The strategy of the proof is to show that if

$$
0=\liminf _{t \rightarrow \infty}|U(t)|<\limsup _{t \rightarrow \infty}|U(t)|,
$$

then the $\omega$-limit set contains a point that is not an equilibrium. By a linear change of variables, we may write $U$ as $(u, v, w) \in \mathbf{R}^{k} \times \mathbf{R}^{l} \times \mathbf{R}^{m}$ so the system may be written

$$
\begin{aligned}
\dot{u} & =A_{+} u+g_{+}(u, v, w), \\
\dot{v} & =A_{-} v+g_{-}(u, v, w), \\
\dot{w} & =g_{0}(u, v, w),
\end{aligned}
$$

where for some $M>0, A_{+}$and $A_{-}$satisfy

$$
u^{T} A_{+} u \geq M|u|^{2}, \quad v^{T} A_{-} v \leq-M|v|^{2} \quad \text { for all } u \in \mathbf{R}^{k}, v \in \mathbf{R}^{l},
$$

and $g=\left(g_{+}, g_{-}, g_{0}\right)$ is $C^{1}$ with $g(0)=0, D g(0)=0$. By the implicit function theorem, the manifold may be parametrized locally for $\max (|u|,|v|,|w|)<$ $\delta_{0}$ by $w \mapsto\left(\alpha_{+}(w), \alpha_{-}(w), w\right)$, where $\alpha_{+}, \alpha_{-}$are $C^{1}$ with $\alpha_{ \pm}(0)=0$, $D \alpha_{ \pm}(0)=0$.

When $U=(u, v, w)$ lies near 0 , we may define

$$
\phi_{+}=u-\alpha_{+}(w), \quad \phi_{-}=v-\alpha_{-}(w) .
$$

Solutions of (14) near zero then satisfy

$$
\begin{aligned}
\dot{\phi}_{+} & =A_{+} \phi_{+}+h_{+}\left(\phi_{+}, \phi_{-}, w\right), \\
\dot{\phi}_{-} & =A_{-} \phi_{-}+h_{-}\left(\phi_{+}, \phi_{-}, w\right), \\
\dot{w} & =h_{0}\left(\phi_{+}, \phi_{-}, w\right),
\end{aligned}
$$


where

$$
\begin{aligned}
h_{ \pm}\left(\phi_{+}, \phi_{-}, w\right) & =g_{+}\left(\phi_{+}+\alpha_{+}, \phi_{-}+\alpha_{-}, w\right)-g\left(\alpha_{+}, \alpha_{-}, w\right) \\
& -D \alpha_{ \pm}(w) \cdot g_{0}\left(\phi_{+}+\alpha_{+}, \phi_{-}+\alpha_{-}, w\right), \\
h_{0}\left(\phi_{+}, \phi_{-}, w\right)= & g_{0}\left(\phi_{+}+\alpha_{+}, \phi_{-}+\alpha_{-}, w\right) .
\end{aligned}
$$

Then $h=\left(h_{+}, h_{-}, h_{0}\right)$ satisfies $h(0,0, w)=0$. Also, $h_{0}$ is $C^{1}$, and $h_{+}, h_{-}$ are $C^{1}$ functions of $\phi_{+}, \phi_{-}$. As a consequence of Taylor's theorem with integral form of remainder, there is a function $\gamma(s) \rightarrow 0$ as $s \rightarrow 0$ such that we have the bound

$$
\left|h\left(\phi_{+}, \phi_{-}, w\right)\right| \leq \gamma(|U|)\left(\left|\phi_{+}\right|+\left|\phi_{-}\right|\right) .
$$

From this estimate it follows that when $\left|\phi_{+}\right|,\left|\phi_{-}\right|,|w|$ are respectively nonzero, we have the estimates

$$
\begin{aligned}
\left|\phi_{+}\right|^{\bullet} & =\phi_{+}^{T} \dot{\phi}_{+} /\left|\phi_{+}\right| \geq(M-\gamma)\left|\phi_{+}\right|-\gamma_{-}\left|\phi_{-}\right|, \\
\left|\phi_{-}\right|^{\bullet} & =\phi_{-}^{T} \dot{\phi}_{-} /\left|\phi_{-}\right| \leq-(M-\gamma)\left|\phi_{-}\right|+\gamma\left|\phi_{+}\right|, \\
|w|^{\bullet} & =w^{T} \dot{w} /|w| \leq \gamma\left(\left|\phi_{+}\right|+\left|\phi_{-}\right|\right),
\end{aligned}
$$

where $\gamma=\gamma(|U|)$.

For $\delta>0$ sufficiently small, define the ball

$$
B(\delta)=\left\{U \in \mathbf{R}^{N} \mid \max \left(\left|\phi_{+}\right|,\left|\phi_{-}\right|,|w|\right) \leq \delta\right\} .
$$

As $\delta \rightarrow 0$, the supremum of $\gamma(|U|)$ over $B(\delta)$ tends to zero. Note that for $\delta$ small, the cone $\left|\phi_{+}\right| \leq\left|\phi_{-}\right|$is locally positively invariant in $B(\delta)$, since $\left|\phi_{+}\right|=\left|\phi_{-}\right| \neq 0$ implies, by (19),

$$
\left(\left|\phi_{+}\right|-\left|\phi_{-}\right|\right)^{\bullet} \geq 2(M-2 \gamma)\left|\phi_{+}\right|>0 .
$$

Suppose that at some time $t_{0}$ we have $U\left(t_{0}\right) \in B(\delta)$. By (14) we may suppose that eventually $U(t)$ leaves $B(2 \delta)$. I claim that $\max \left(\left|\phi_{+}\right|,\left|\phi_{-}\right|\right)=2 \delta$ when this happens. First, at time $t_{0}$ either $\left|\phi_{+}\right|<\left|\phi_{-}\right|$or $\left|\phi_{+}\right| \geq\left|\phi_{-}\right|$. Consider the first case. As long as $\left|\phi_{+}\right| \leq\left|\phi_{-}\right| \neq 0$, we have

$$
\left|\phi_{-}\right|^{\bullet} \leq-(M-2 \gamma)\left|\phi_{-}\right|<0, \quad\left(4|w|+\left|\phi_{-}\right|\right)^{\bullet} \leq-\left(M-10_{\gamma}\right)\left|\phi_{-}\right|<0 .
$$

Hence if $\left|\phi_{+}(t)\right| \leq\left|\phi_{-}(t)\right|$ for $t_{0} \leq t \leq t_{1}$, we have

$$
|w(t)| \leq\left|w\left(t_{0}\right)\right|+\left|\phi_{-}\left(t_{0}\right)\right| / 4 \leq 5 \delta / 4 .
$$

Since the solution leaves $B(2 \delta)$, we will have $\left|\phi_{+}\left(t_{1}\right)\right| \geq\left|\phi_{-}\left(t_{1}\right)\right|$ eventually, i.e., the second case. In this case,

$$
\left(4|w|+\left|\phi_{+}\right|\right)^{\bullet} \leq-(M-10 \gamma)\left|\phi_{+}\right|<0,
$$

so $|w(t)| \leq\left|w\left(t_{1}\right)\right|+\left|\phi_{+}(t)\right| / 4$. Putting the two cases together we find that if $U\left(t_{0}\right) \in B(\delta)$ and $U(t) \in B(2 \delta)$ for $t_{0}<t<T$, then

$$
|w(t)| \leq\left|w\left(t_{0}\right)\right|+\left|\phi_{+}(t)\right| / 4+\left|\phi_{-}\left(t_{0}\right)\right| / 4<7 \delta / 4 .
$$

This proves the claim.

Now as a consequence of assumption (14), we may extract a subsequence $t_{n} \rightarrow \infty$ such that $U\left(t_{n}\right)$ converges and $\max \left(\left|\phi_{+}\left(t_{n}\right)\right|,\left|\phi_{-}\left(t_{n}\right)\right|\right)=2 \delta$ for all $n$. Denoting the limit by $U_{*}=\left(u_{*}, v_{*}, w_{*}\right)$, we have 


$$
\max \left(\left|u_{*}-\alpha_{+}\left(w_{*}\right)\right|,\left|v_{*}-\alpha_{-}\left(w_{*}\right)\right|\right)=2 \delta \neq 0,
$$

so that $U_{*}$ is not an equilibrium point. This proves Theorem 2 .

\section{ACKNOWLEDGMENTS}

Thanks go to my host M. Rascle at the Université de Nice, where this work was done.

\section{REFERENCES}

[AB] G. Andrews and J. M. Ball, Asymptotic behaviour and changes of phase in one-dimensional nonlinear viscoelasticity, J. Differential Equations 44 (1982), 306-341.

[HM] J. K. Hale and P. Massatt, Asymptotic behavior of gradient-like systems, Dynamical Systems II (Gainesville FL. 1981) pp. 85-101. (A. R. Bednarek and L. Cesari, eds.), Academic Press, 1982.

[NPT] J. A. Nohel, R. L. Pego, and A. E. Tzavaras, Stability of discontinuous steady states in shearing motions of a non-Newtonian fluid, Proc. Roy. Soc. Edinburgh Sect. A 115 (1990), 39-59.

[NP] A. Novick-Cohen and R. L. Pego, Stable patterns in a viscous diffusion equation, Trans. Amer. Math. Soc. 324 (1991) 331-351.

[P] R. L. Pego, Phase transitions in one-dimensional nonlinear viscoelasticity: admissibility and stability, Arch. Rational Mech. Anal. 97 (1987), 353-394.

Department of Mathematics, University of Maryland, College Park, Maryland 20742 